\title{
Communication \\ Dispersion Optimization of Silicon Nitride Waveguides for Efficient Four-Wave Mixing
}

\author{
Yaping Hong ${ }^{1,+}$, Yixiao Hong ${ }^{2,+}$, Jianxun Hong ${ }^{1, *}{ }^{\mathbb{C}}$ and Guo-Wei $\mathrm{Lu}^{3,+}$ \\ 1 School of Information Engineering, Wuhan University of Technology, Wuhan 430070, China; \\ hyping@whut.edu.cn \\ 2 School of Electrical and Electronic Engineering, Hubei University of Technology, Wuhan 430068, China; \\ yxhong@hbut.edu.cn \\ 3 Division of Computer Engineering, The University of Aizu, Fukushima 965-8580, Japan; \\ guoweilu@u-aizu.ac.jp \\ * Correspondence: jxhong@whut.edu.cn \\ + These authors contributed equally to this work.
}

check for updates

Citation: Hong, Y.; Hong, Y.; Hong, J.; Lu, G.-W. Dispersion Optimization of Silicon Nitride Waveguides for Efficient Four-Wave Mixing. Photonics 2021, 8, 161. https://doi.org/ $10.3390 /$ photonics 8050161

Received: 17 April 2021

Accepted: 10 May 2021

Published: 11 May 2021

Publisher's Note: MDPI stays neutral with regard to jurisdictional claims in published maps and institutional affiliations.

Copyright: (C) 2021 by the authors Licensee MDPI, Basel, Switzerland. This article is an open access article distributed under the terms and conditions of the Creative Commons Attribution (CC BY) license (https:// creativecommons.org/licenses/by/ $4.0 /)$

\begin{abstract}
Silicon nitride waveguides have emerged as an excellent platform for photonic applications, including nonlinear optical signal processing, owing to their relatively high Kerr nonlinearity, negligible two photon absorption, and wide transparent bandwidth. In this paper, we propose an effective approach using 3D finite element method to optimize the dispersion characteristics of silicon nitride waveguides for four-wave mixing (FWM) applications. Numerical studies show that a flat and low dispersion profile can be achieved in a silicon nitride waveguide with the optimized dimensions. Near-zero dispersion of $1.16 \mathrm{ps} / \mathrm{km} / \mathrm{nm}$ and $0.97 \mathrm{ps} / \mathrm{km} / \mathrm{nm}$ at a wavelength of $1550 \mathrm{~nm}$ are obtained for plasma-enhanced chemical vapor deposition (PECVD) and low-pressure chemical vapor deposition (LPCVD) silicon nitride waveguides, respectively. The fabricated micro-ring resonator with the optimized dimensions exhibits near-zero dispersion of -0.04 to $-0.1 \mathrm{ps} / \mathrm{m} / \mathrm{nm}$ over a wavelength range of $130 \mathrm{~nm}$ which agrees with the numerical simulation results. FWM results show that near-zero phase mismatch and high conversion efficiencies larger than $-12 \mathrm{~dB}$ using a low pump power of $0.5 \mathrm{~W}$ in a $13-\mathrm{cm}$ long silicon nitride waveguide are achieved.
\end{abstract}

Keywords: four-wave mixing; dispersion; phase matching; silicon nitride waveguide; conversion efficiency

\section{Introduction}

With the rapid development of the modern optical communication, the technology of integrated photonic devices is continuously developing. Four-wave mixing (FWM), as an important third-order nonlinear optical process, can be used for wavelength conversion and optical signal processing in integrated waveguides, and has received intense investigations on different platform [1]. Materials such as silicon, silicon dioxide, silicon-rich silica, silicon nitride, and nonlinear polymer have been used for FWM applications. The refractive index of silicon is higher, and the optical band gap $(1.1 \mathrm{eV})$ is smaller. However, the conversion efficiency and signal processing speed of the FWM effect in pure silicon are limited by the nonlinear loss (two photon absorption induced free carrier absorption) [2,3]. Silicon dioxide has a smaller refractive index, but the nonlinear index is very low $\left(2.7 \times 10^{-20} \mathrm{~m}^{2} / \mathrm{W}\right.$ at $1550 \mathrm{~nm}$ ), thus it needs a long waveguide to achieve high nonlinear effect. As an alternative choice, silicon nitride waveguides have a moderately high index contrast, a relatively high Kerr nonlinearity, and a negligible two photon absorption around telecom wavelengths, which makes it a potential candidate for photonic integration platforms [4-7]. Moreover, silicon nitride has a wide transparent window ranging from visible to near infrared wavelengths [8]. As a consequence, excellent silicon nitride-based nonlinear optical applications have been demonstrated, including optical frequency combs $[9,10]$, 
broadband supercontinuum generation [11], self-phase modulation [12], and FWM-based wavelength conversion [13]. Usually, silicon nitride thin films can be prepared by PECVD or low-LPCVD [4].

The dispersion properties of the waveguides are significant for the ultrafast nonlinear applications [14-17]. The lack of dispersion management might lower the wavelength conversion efficiency and limit the tuning range of the FWM process. In principle, near-zero dispersion over a wavelength range is required to achieve phase matching for high FWM conversion efficiencies $[18,19]$. Note that other third-order nonlinear applications, such as optical frequency combs, broadband supercontinuum generation, self-phase modulation, and cross-phase modulation also require dispersion engineering for phase matching. Both the material dispersion and waveguide dispersion contribute to the total dispersion. The waveguide dispersion can be tailored by controlling the structure parameters of the waveguide $[10-12,17]$. The phase matching conditions are mainly dominated by second-order dispersion, which is usually described by the dispersion coefficient $D$.

In this paper, systematic analyses on the dispersion properties of PECVD and LPCVD silicon nitride channel waveguides are conducted, and the geometric parameters of the waveguides to obtain near-zero dispersions for phase matching are optimized. The fabricated silicon nitride micro-ring resonator with optimized parameter shows near-zero dispersion of $-0.07 \mathrm{ps} / \mathrm{m} / \mathrm{nm}$ at $1550 \mathrm{~nm}$ and a flat dispersion profile over a $130 \mathrm{~nm}$ wavelength range. FWM results show that the optimized silicon nitride waveguide exhibits a high conversion efficiency larger than $-12 \mathrm{~dB}$.

\section{Waveguide Dispersion Analysis Principle}

The silicon nitride channel waveguide is designed on silicon substrate with a $\mathrm{SiO}_{2}$ claddings. The thicknesses of the bottom and top claddings are $3 \mu \mathrm{m}$. The width and the thickness of the core are denoted by $w$ and $t$, respectively. The relationships between the effective refractive index and the group refractive index and the dispersion coefficient can be expressed by following equations [20]:

$$
\begin{gathered}
n_{g}=n_{\mathrm{eff}}-\lambda \frac{d n_{\mathrm{eff}}}{d \lambda} n_{g}=n_{\mathrm{eff}}-\lambda \frac{d n_{\mathrm{eff}}}{d \lambda} \\
D(\lambda)=\frac{1}{c} \frac{d n_{g}}{d \lambda}=-\frac{\lambda}{c} \frac{d^{2} n_{\mathrm{eff}}}{d \lambda^{2}}=-\frac{2 \pi c}{\lambda^{2}} \beta_{2}
\end{gathered}
$$

where $n_{\text {eff }}$ is the effective refractive index, $n_{g}$ is the group refractive index, $\lambda$ is the wavelength, $c$ is the speed of the light, $\beta_{2}$ is the group velocity dispersion. The effective refractive index of the waveguide depends on the waveguide width. thickness and the material refractive index. By analyzing the spectrum of $n_{\text {eff }}$, the group refractive index and dispersion of the waveguide can be extracted according to Equations (1) and (2).

\section{Results and Discussion}

\subsection{Waveguide Dispersion Optimiation}

The PECVD silicon nitride is deposited at temperature of $350^{\circ} \mathrm{C}$ with a gas flow ratio of $\mathrm{SiH}_{4}: \mathrm{NH}_{3}: \mathrm{N}_{2}=6: 3: 189 \mathrm{sccm}$. The LPCVD silicon nitride is prepared by using dichlorosilane (DCS) and ammonia $\left(\mathrm{NH}_{3}\right)$ at $800{ }^{\circ} \mathrm{C}$ with a DCS to $\mathrm{NH}_{3}$ ratio of 0.3 [17]. The material refractive indices are shown in Figure 1. The data will be used in effective refractive index and dispersion calculation. The CVD deposited silicon nitride film can be silicon-rich or nitrogen-rich. Due to the different deposition temperature and gas ratio, there is a relatively large difference between refractive indices of silicon nitride films prepared by PECVD and LPCVD [21,22]. 


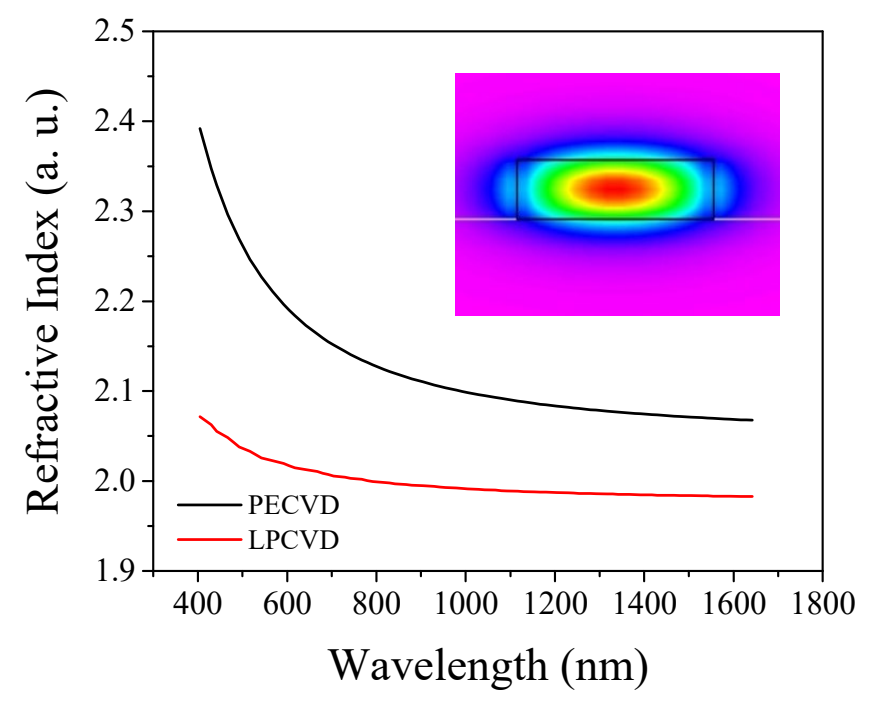

Figure 1. The refractive index of silicon nitride prepared by PECVD and LPCVD. The insert is the optical field distribution of the fundamental TE mode, here, the width, thickness, and refractive index of silicon nitride are $710 \mathrm{~nm}, 1250 \mathrm{~nm}$ and 2.0, respectively.

\subsubsection{Dispersion of Silicon Nitride Thin Film Waveguide of PECVD}

In this study, only the dispersion of the fundamental TE mode is studied because TM modes become TE modes if the waveguide rotates $90^{\circ}$. The filed distribution of the silicon nitride channel waveguide is calculated by using 3D finite element method (3D-FEM). The insert in Figure 1 shows the field distribution of the fundamental mode of the PECVD silicon nitride waveguide at $1550 \mathrm{~nm}$ with a width of $710 \mathrm{~nm}$ and a thickness of $1250 \mathrm{~nm}$. It can be seen that the optical power is well confined in the core of the waveguide.

The effective refractive index of the mode is obtained from the mode calculation. Figure 2 shows the effective refractive index and dispersion of the waveguides with different width and thickness at the wavelength of $1550 \mathrm{~nm}$. Note that the dispersion includes the waveguide dispersion and material dispersion. It can be seen that the effective refractive index of the waveguide increases with the width and thickness increasing because more power is confined in the waveguide core while the cross-section is larger.
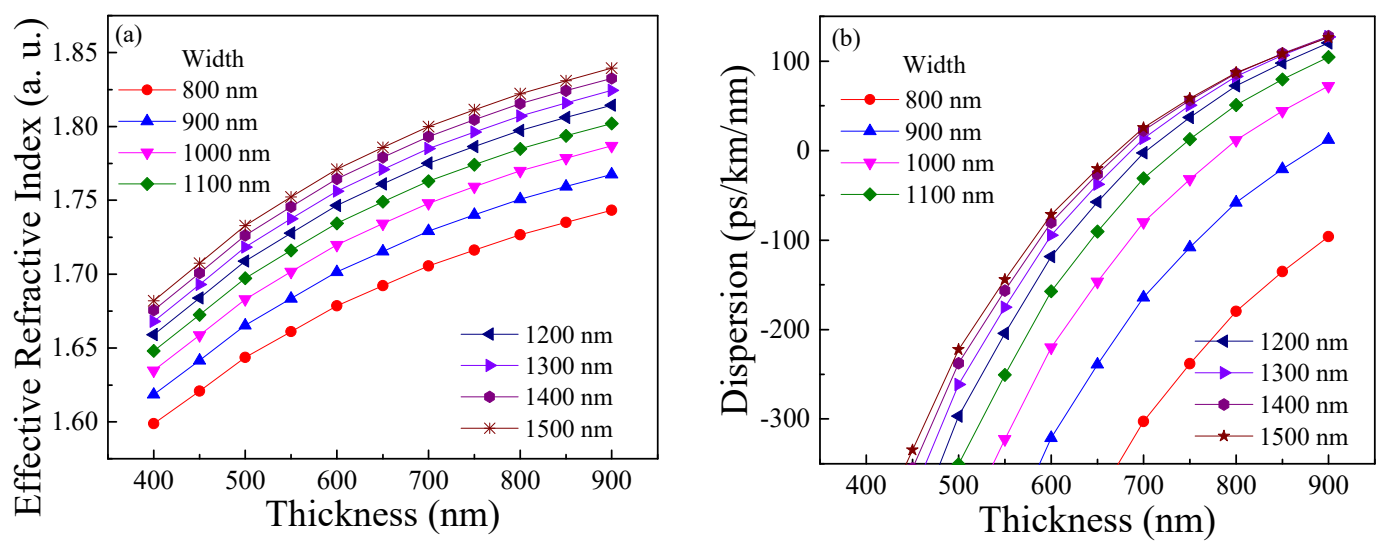

Figure 2. (a) The effective refractive index, and (b) the dispersion of PECVD silicon nitride waveguide with different widths and thicknesses.

The dispersion increases with the width and thickness increasing. The dispersion increases slowly with the width while the width is larger than $1200 \mathrm{~nm}$. This is because the optical mode distribution is well confined in the waveguide core and weakly affected by the width increase. For the same reason, the thickness dependence of the dispersion will exhibit a similar characteristic. For TE mode, the dispersion changes faster with the 
thickness than with the width. However, the optimization of the width is easier than that of the thickness because it can be simply realized by designing the photolithograph pattern. Moreover, a very large thickness of the silicon nitride film will add much difficulty to the dry etching of the device. Therefore, a tradeoff between the device fabrication and the dispersion engineering should be made. While the width changing is not enough to get a target dispersion especially an anomalous dispersion $(D>0)$, the thickness should be changed during experimental optimization.

According to Figure $2 b$, near-zero dispersions can be easily obtained while the thickness is around $700 \mathrm{~nm}$ and the width is around $1200 \mathrm{~nm}$. We choose the waveguide parameters as $t=710 \mathrm{~nm}$ and $w=1250 \mathrm{~nm}$ which offers an obvious fabrication tolerance. Thus, the dispersion of the selected dimension is slightly larger than zero which might satisfy the phase matching requirement of FWM. It should be noted that the core dimension of the waveguide does not meet the condition of single-mode propagation. However, the power of the high order mode distributes to the edge and attenuates very fast due to transmission loss. Therefore, the high order mode dispersion of the waveguide can be ignored and the dimension supports effective nonlinear interaction between the fundamental mode and the materials. The spectrum of the dispersion of the selected waveguide is shown in Figure 3. The dispersion is near zero $( \pm 20 \mathrm{ps} / \mathrm{km} / \mathrm{nm})$ over a $100 \mathrm{~nm}$ wavelength range around $1550 \mathrm{~nm}$, and is close to zero $(\sim 1.16 \mathrm{ps} / \mathrm{km} / \mathrm{nm})$ at $1550 \mathrm{~nm}$, which can offer an excellent phase matching condition.

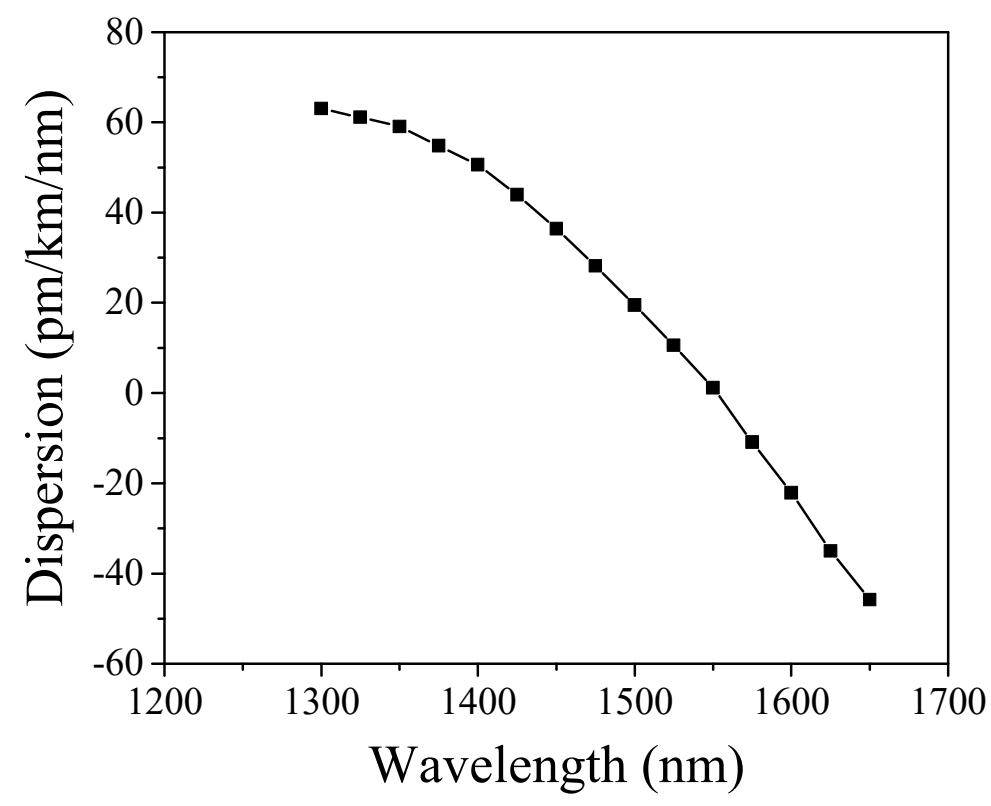

Figure 3. The dispersion as a function of wavelength of PECVD silicon nitride waveguide with optimized thickness of $710 \mathrm{~nm}$ and width of $1250 \mathrm{~nm}$ to get a near-zero dispersion around the wavelength of $1.55 \mu \mathrm{m}$.

\subsubsection{Dispersion of Silicon Nitride Thin Film Waveguide of LPCVD}

In this section, we analyze the dispersion of channel waveguides of silicon nitride deposited by using LPCVD. Figure 4a shows the dispersion of the waveguide with different width and thickness at the wavelength of $1550 \mathrm{~nm}$. It can be seen that the dispersion increases with the width and thickness. The zero dispersion point can be found on the curve of $w=1100 \mathrm{~nm}$. Therefore, the waveguide with thickness of $790 \mathrm{~nm}$ and width of $1100 \mathrm{~nm}$ was selected, which are close to the optimized parameters of PECVD silicon nitride waveguide. The dispersion spectrum of the optimized waveguide is shown in Figure $4 \mathrm{~b}$. It shows flat and low dispersion over a wide wavelength range, and a near-zero dispersion of $0.97 \mathrm{ps} / \mathrm{km} / \mathrm{nm}$ at the wavelength of $1550 \mathrm{~nm}$ is obtained. 

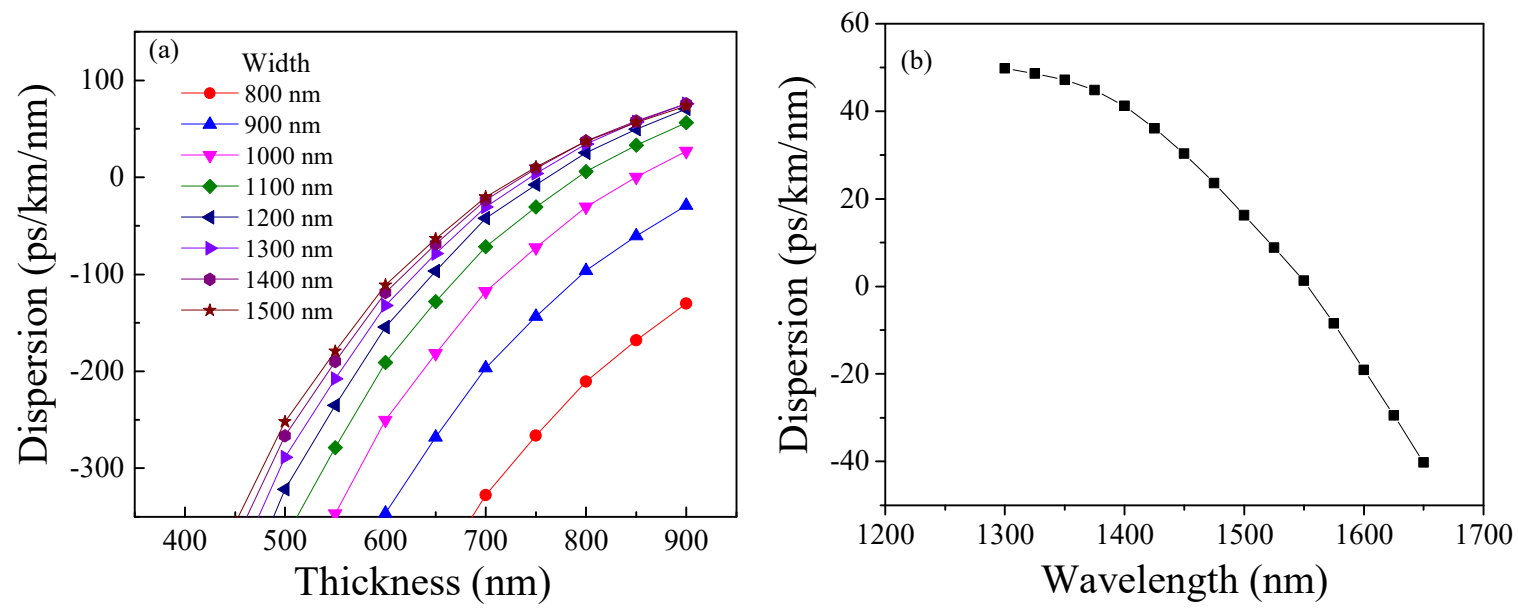

Figure 4. The dispersion of LPCVD silicon nitride waveguide (a) with different widths and thicknesses, and (b) with an optimized thickness of $790 \mathrm{~nm}$ and width of $1100 \mathrm{~nm}$.

Compared Figures 2 and 4, it can be seen that PECVD and LPCVD silicon nitride waveguides exhibit similar dispersion characteristics. Even though the refractive index characteristics are different, near-zero dispersion can be obtained to meet the phase matching for FWM, in which the thickness is in the range of $700 \mathrm{~nm}$ to $800 \mathrm{~nm}$ and the width is in the range of $1100 \mathrm{~nm}$ to $1300 \mathrm{~nm}$. If the dispersion is optimized for TM mode in silicon nitride waveguide, a larger waveguide thickness $(>1 \mu \mathrm{m})$ will be required because of the rotational relationship between TM and TE modes. The larger thickness might add more difficulty to the film deposition and cause more side wall roughness during etching [4].

\subsection{Waveguide Fabrication and Experimental Characterization}

The dispersion of waveguides can be experimentally analyzed by measuring the spectrum of an interference waveguide structure such as an MZI interferometer or a micro-ring resonator. Therefore, we fabricate a micro-ring resonator on a standard 4-inch silicon wafer with a $3 \mu \mathrm{m}$-thick $\mathrm{SiO}_{2}$ layer according to the optimized parameters. The radium of the ring, the waveguide width, the gap between the bus waveguide and the micro-ring are $250 \mu \mathrm{m}, 1250 \mathrm{~nm}$ and $150 \mathrm{~nm}$, respectively. The $710 \mathrm{~nm}$-thick silicon nitride film is firstly deposited on the wafer at a temperature of $350{ }^{\circ} \mathrm{C}$ with a gas flow ratio of $\mathrm{SiH}_{4}: \mathrm{NH}_{3}: \mathrm{N}_{2}=6: 3: 189 \mathrm{sccm}$ by using the PECVD (SAMCO, PD-220NL). A $500 \mathrm{~nm}$-thick positive photoresist ARP6200 is then spin-coated on the wafer at the speed of $1500 \mathrm{rpm}$. After baking at $150{ }^{\circ} \mathrm{C}$ for $1 \mathrm{~min}$, the waveguide structure is then patterned by electronic beam lithography (EBL, Elionix, ELS-G100) and etched $710 \mathrm{~nm}$ onto the silicon nitride layer by ion coupling plasma (ICP, SAMCO, RIE-400iPB) with the gas of $\mathrm{CHF}_{3}$. The gap flow, bias power and ICP power are $6 \mathrm{sccm}, 50 \mathrm{~W}$, and $50 \mathrm{~W}$, respectively. After removing the photoresist, a $3 \mu \mathrm{m}$-thick $\mathrm{SiO}_{2}$ layer is then deposited on the sample as a top cladding at $150{ }^{\circ} \mathrm{C}$ by using liquid source chemical vapor deposition (LSCVD, SAMCO, PD100ST) [23]. Figure 5 illustrates the schematic illustration, micrograph and the SEM image in the coupling zone of the micro-ring resonator. The light coupling was conducted by using waveguide grating couplers which offer excellent design and application flexibilities [24]. The grating couplers are optimized at $1550 \mathrm{~nm}$ and the coupling efficiency is about $-3.5 \mathrm{~dB}$. The fabricated device agrees well with the design. 

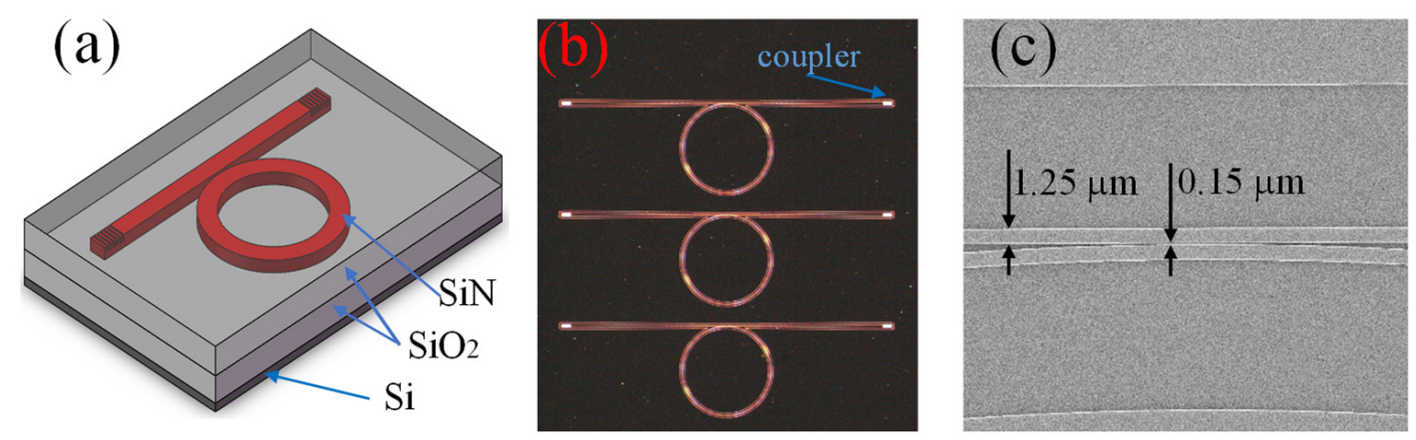

Figure 5. (a) The schematic illustration, (b) micrograph, and (c) SEM image in the coupling region of the micro-ring resonators.

In order to measure the transmission spectrum of the micro-ring resonator, light from a tunable laser (SANTEC TSL550) is pumped into the input waveguide. The wavelength is swept over a range of $130 \mathrm{~nm}$ and the spectrum is recorded by an optical power meter (SANTEC MPM200) with 1 pm wavelength resolution and shown in Figure 6a. The insert shows the experimental setup. According to the spectrum data, the free spectral range (FSR) is extracted and shown in Figure 6b. It can be seen that FSR increases gradually with the wavelength. Note that, the transmission loss of the waveguide is estimated to be $1.82 \mathrm{~dB} / \mathrm{cm}$ by measuring the fiber-to fiber losses of waveguides with different lengths.
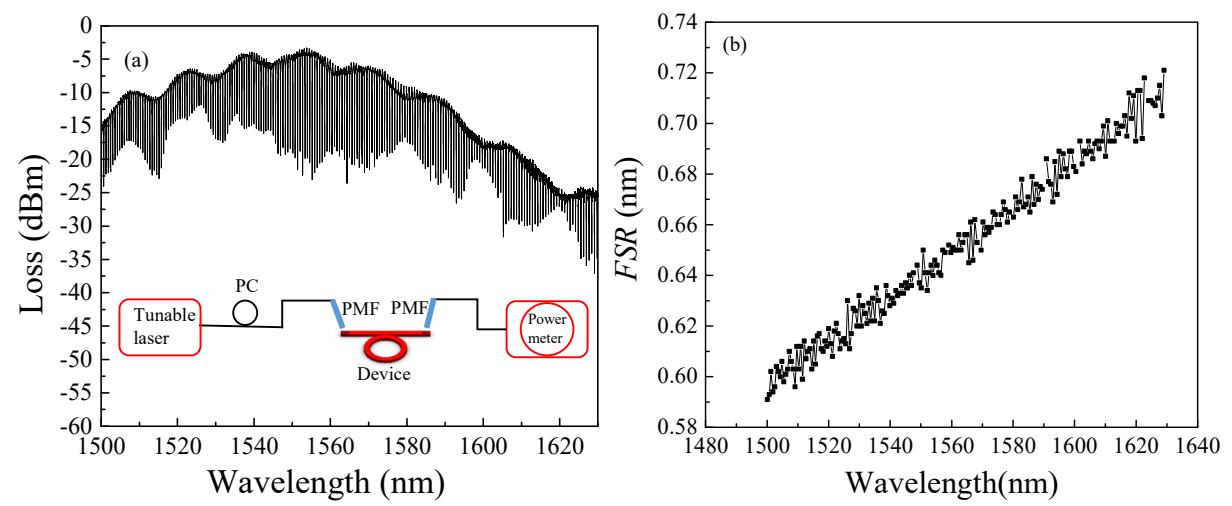

Figure 6. (a) The measured transmission spectrum and (b) the FSR of the silicon nitride microring resonator. The insert is the experimental setup. PC: polarization controller, PMF: polarization maintain fiber.

The relationship between the group refractive index and FSR can be written as [25]:

$$
n_{g}=\frac{\lambda^{2}}{2 \pi R F S R}
$$

where $R$ is the radius of the ring. According to Equation (3), the group refractive index is calculated and shown in Figure 7a. The red line is the polynomial fitting of the data. By substituting the fitting into Equation (2), the dispersion is calculated and shown in Figure $7 \mathrm{~b}$. It shows a flat and low dispersion profile within -0.04 to $-0.1 \mathrm{ps} / \mathrm{m} / \mathrm{nm}$ from wavelength $1500 \mathrm{~nm}$ to $1630 \mathrm{~nm}$ (a range of $130 \mathrm{~nm}$ ). Near-zero dispersion of $-0.07 \mathrm{ps} / \mathrm{m} / \mathrm{nm}$ at the wavelength of $1550 \mathrm{~nm}$ is achieved. The dispersion decreases with the wavelength which is similar to the simulation results shown in Figures 3 and 4 . The wavelength dependence of the dispersion is near linear in Figure $7 \mathrm{~b}$, which is a bit different to those shown in Figures 3 and 4 at short wavelengths. It can be explained that the material dispersion which is very linear in the wavelength range as shown in Figure 1 contributes much to the total dispersion of the fabricated device due to the relatively small waveguide size of the micro-ring resonator. 

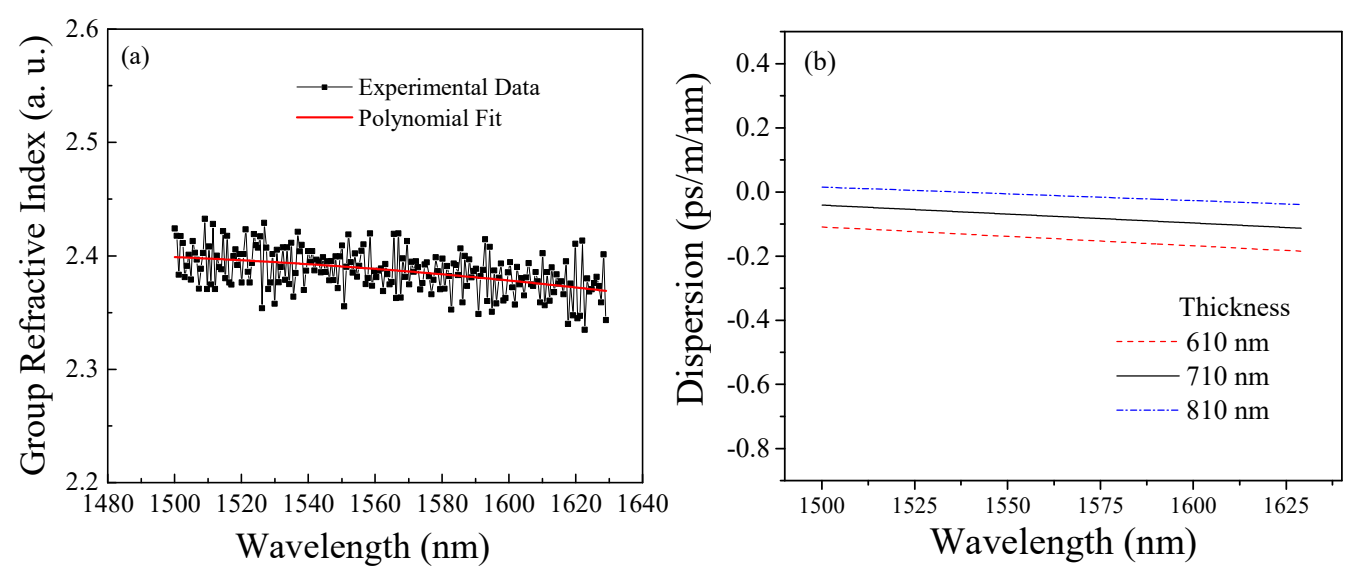

Figure 7. (a) The calculated group refractive index, and (b) dispersion of the silicon nitride microring resonator.

Dispersions of the waveguides with thicknesses of $610 \mathrm{~nm}$ and $810 \mathrm{~nm}$ are also measured and shown in Figure $7 \mathrm{~b}$. Simulation and experiment results show that nearzero anomalous dispersion $(D>0)$ can be realized by tailoring the dimensions of the waveguides. Sometimes, anomalous dispersion is required for phase matching $[10,18]$. Especially in the case of large loss existing, the anomalous dispersion can compensate the phase mismatching caused by the linear and nonlinear losses. As demonstrated before, only increasing the width might be incapable of resulting in an anomalous dispersion if the thickness is small. In order to get a near-zero anomalous dispersion, the thickness of $810 \mathrm{~nm}$ or larger values is suitable.

\subsection{Phase Mismatch and Conversion Efficiency of the Waveguide}

The phase mismatch of the waveguide for FWM can be expressed as [26-28]:

$$
\kappa=\Delta \beta+2 \gamma P_{P}=\beta_{s}+\beta_{c}-2 \beta_{p}+2 \gamma P_{P} \approx \beta_{2}\left(\omega_{s}-\omega_{p}\right)^{2}+2 \gamma P_{P}
$$

where $\beta_{p, s, c}$ and $\omega_{p, s, c}$ are the wavenumbers and frequencies of the interaction optical waves, $P_{p}$ is the pump power. the first and second terms, $\Delta \beta$ and $2 \gamma P_{p}$, are referred to as linear and nonlinear phase mismatch, respectively. $\gamma$ is the waveguide nonlinear coefficient and is defined as [29]:

$$
\gamma=\frac{2 \pi n_{2}}{\lambda A_{\mathrm{eff}}}
$$

where $n_{2}$ is the nonlinear refractive index, $A_{\text {eff }}$ is effective mode area of the waveguide. According to the mode distribution, $\gamma$ is calculated to be $1.19 \mathrm{~m}^{-1} \mathrm{~W}^{-1}$ at $1550 \mathrm{~nm}$ by assuming $n_{2}=2.6 \times 10^{-19} \mathrm{~m}^{2} \mathrm{~W}^{-1}$ for silicon nitride.

The phase mismatch depends on the dispersion, frequency difference, waveguide nonlinear coefficient and input pump power. Because the value of $\gamma$ is positive and not too large, a near-zero dispersion especially anomalous dispersion can make the phase mismatch close to zero. Figure 8 shows the phase mismatch of the waveguide with different input pump power. The near-zero phase mismatch $\left( \pm 0.2 \mathrm{~cm}^{-1}\right)$ is obtained over a $30 \mathrm{~nm}$ wavelength range near the pump wave, which is attributed to the optimized nearzero anomalous dispersion of the silicon nitride waveguide. It also shows a weak phase mismatch dependence on the pump power, which means that the conversion efficiency can be effectively improved by increasing the pump power. 


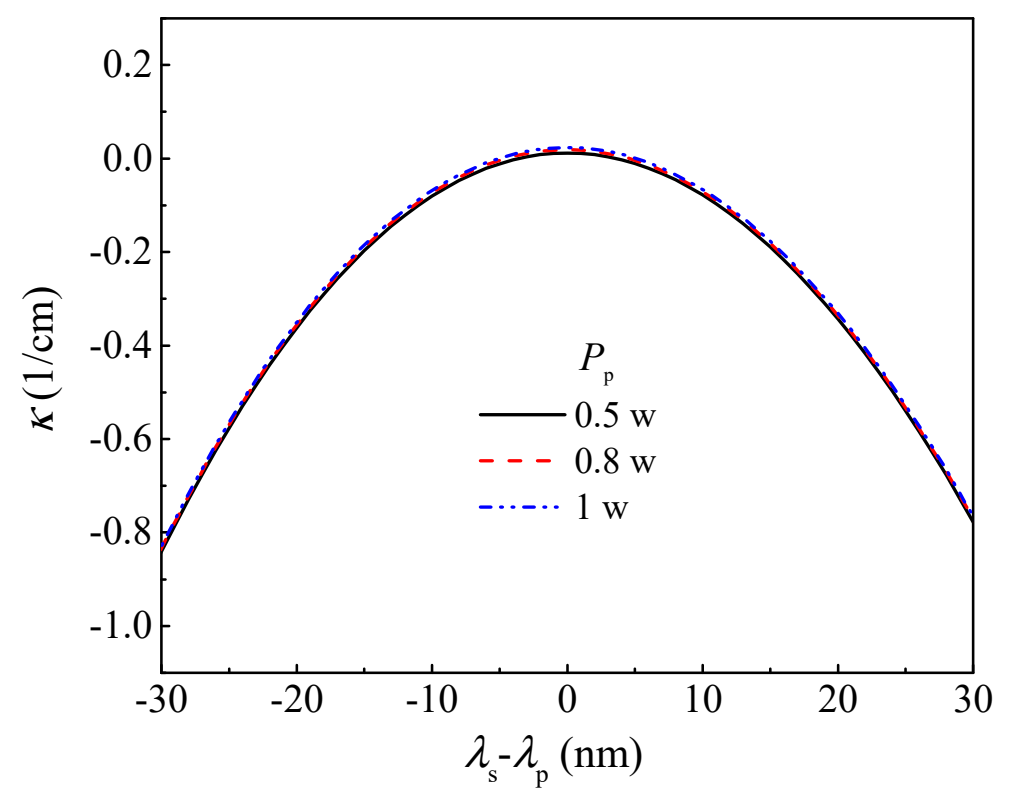

Figure 8. The calculated phase mismatch of the PECVD silicon nitride waveguide at different pump power. Here, the pump wavelength is $1550 \mathrm{~nm}$. The width and height of the waveguide are $1250 \mathrm{~nm}$ and $710 \mathrm{~nm}$, respectively.

The coupled equations of the FWM process can be expressed as [27,30]:

$$
\begin{gathered}
\frac{d A_{p}}{d z}=-\frac{1}{2} \alpha A_{p}+j \gamma\left|A_{p}\right|^{2} A_{p} \\
\frac{d A_{s}}{d z}=-\frac{1}{2} \alpha A_{s}+2 j \gamma\left|A_{p}\right|^{2} A_{s}+j \gamma A_{i}^{*} A_{p}^{2} \exp (-j \Delta \beta z) \\
\frac{d A_{i}}{d z}=-\frac{1}{2} \alpha A_{i}+2 j \gamma\left|A_{p}\right|^{2} A_{i}+j \gamma A_{s}^{*} A_{p}^{2} \exp (-j \Delta \beta z)
\end{gathered}
$$

where $A_{p, s, c}$ are the amplitude of the interaction optical waves, $\alpha$ is the transmission loss factor, $z$ is the coordinate along the transmission direction. The conversion efficiency (CE) defined as the power ratio of the output idler power to signal power are calculated. Figure 9 shows the $\mathrm{CE}$ dependence on the waveguide length with an input pump power of $0.5 \mathrm{~W}$. It can be seen that $\mathrm{CE}$ as high as $-12 \mathrm{~dB}$ can be obtained with a length of $13 \mathrm{~cm}$. The CE increases slowly while the length is larger than $13 \mathrm{~cm}$ because the accumulated transmission loss in a very long waveguide attenuates the generated idler power. Moreover, the optical power in a very long waveguide changes obviously during transmission due to the nonnegligible loss. The phase mismatch increases with the transmission distance. Therefore, as can be seen in Figure 9, the CE changes more obviously with the input signal wavelength while the length is larger. The $\mathrm{CE}$ decreases with the input signal wavelength decreasing because the wavelength difference between the pump and signal waves enlarges the phase mismatch as shown in Figure 8. 


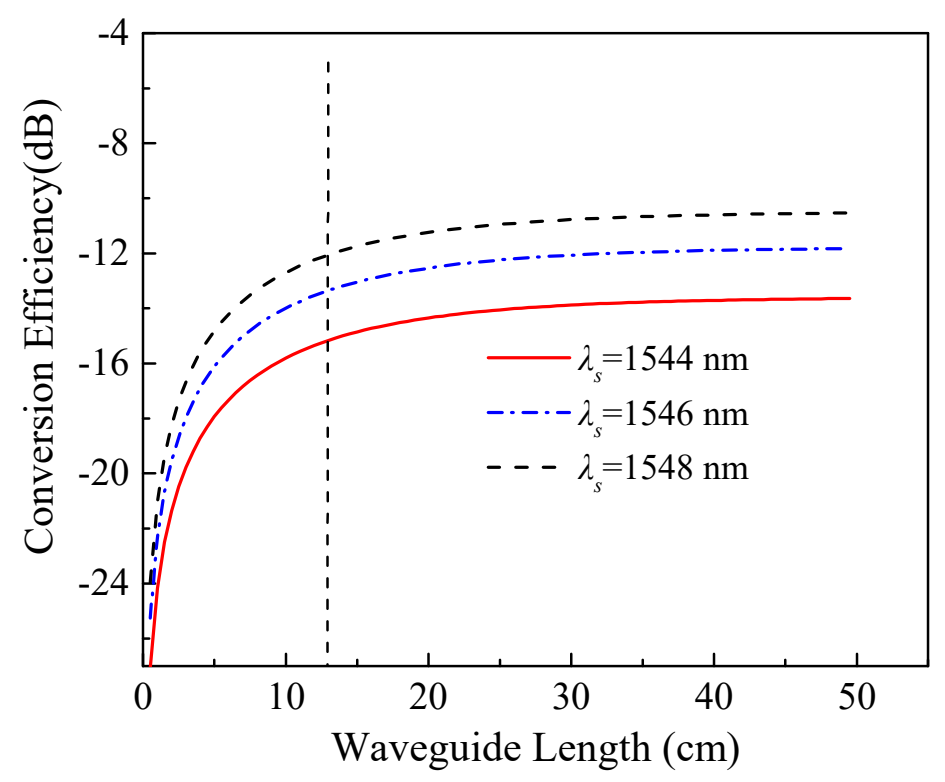

Figure 9. The calculated conversion efficiency of the FWM process of the PECVD silicon nitride waveguide. Here, the pump power and pump wavelength are $0.5 \mathrm{~W}$ and $1550 \mathrm{~nm}$, respectively.

\section{Conclusions}

In this paper, we analyzed the group velocity dispersion of the silicon nitride waveguides. In order to find the near-zero dispersion points for the phase matching of FWMbased application, we optimize the core dimension of the silicon nitride waveguide. The effective refractive index and the dispersion of the waveguide increase with the width and thickness increasing, and the dispersion decreases with the wavelength increasing. The optimum core dimension of the PECVD silicon nitride waveguide is $710 \mathrm{~nm} \times 1250 \mathrm{~nm}$ and the corresponding dispersion is $1.16 \mathrm{ps} / \mathrm{km} / \mathrm{nm}$. The optimized core dimension of the LPCVD silicon nitride waveguide is $790 \times 1100 \mathrm{~nm}$ and the corresponding dispersion is $0.97 \mathrm{ps} / \mathrm{km} / \mathrm{nm}$. Results show that the dimensions of the PECVD and LPCVD silicon nitride for the phase matching of FWM are similar.

The dispersion of a PECVD silicon nitride micro-ring resonator is experimentally characterized with the core dimensions of $t=710 \mathrm{~nm}$ and $w=1250 \mathrm{~nm}$. Results show that a near-zero dispersion of $-0.07 \mathrm{ps} / \mathrm{m} / \mathrm{nm}$ at the wavelength of $1550 \mathrm{~nm}$ is obtained which is consistent with the theoretical results. Owing to the optimized dispersion of the waveguides, near-zero phase mismatch over a wavelength range of $30 \mathrm{~nm}$ is obtained, which ensures high CEs larger than $-12 \mathrm{~dB}$ with a low input pump power of $0.5 \mathrm{~W}$ in a $13 \mathrm{~cm}$ long silicon nitride waveguide.

Author Contributions: Conceptualization: J.H. and G.-W.L.; data curation: Y.H. (Yaping Hong) and J.H.; methodology: J.H.; software: Y.H. (Yaping Hong) and Y.H. (Yixiao Hong); validation: Y.H. (Yaping Hong), J.H., and G.-W.L.; writing—original draft: Y.H. (Yaping Hong) and J.H.; writingreview and editing: Y.H. (Yixiao Hong), J.H., and G.-W.L.; funding acquisition: G.-W.L. All authors have read and agreed to the published version of the manuscript.

Funding: This research received no external funding.

Institutional Review Board Statement: Not applicable.

Informed Consent Statement: Not applicable.

Data Availability Statement: Not applicable.

Conflicts of Interest: The authors declare no conflict of interest. 


\section{References}

1. Helt, L.G.; Liscidini, M.; Sipe, J.E. How Does It Scale? Comparing Quantum and Classical Nonlinear Optical Processes in Integrated Devices. J. Opt. Soc. Am. B 2012, 29, 2199-2212. [CrossRef]

2. Wu, C.; Huang, J.; Ou, D.; Liao, T.; Chiu, Y.; Shih, M.; Lin, Y.; Chu, A.; Lee, C. Efficient Wavelength Conversion with Low Operation Power in a $\mathrm{Ta}_{2} \mathrm{O}_{5}$-Based Micro-Ring Resonator. Opt. Lett. 2017, 42, 4804-4807. [CrossRef]

3. McMillan, J.F.; Yu, M.; Kwong, D.; Wong, C.W. Observation of Four-Wave Mixing in Slow-Light Silicon Photonic Crystal Waveguides. Opt. Express 2010, 18, 15484-15497. [CrossRef]

4. Yakuhina, A.; Kadochkin, A.; Svetukhin, V.; Gorelov, D.; Generalov, S.; Amelichev, V. Investigation of Side Wall Roughness Effect on Optical Losses in a Multimode $\mathrm{Si}_{3} \mathrm{~N}_{4}$ Waveguide Formed on a Quartz Substrate. Photonics 2020, 7, 104. [CrossRef]

5. Moss, D.J.; Morandotti, R.; Gaeta, A.L.; Lipson, M. New CMOS-Compatible Platforms Based on Silicon Nitride and Hydex for Nonlinear Optics. Nat. Photonics 2013, 7, 597-607. [CrossRef]

6. Ikeda, K.; Saperstein, R.E.; Alic, N.; Fainman, Y. Thermal and Kerr Nonlinear Properties of Plasma-Deposited Silicon Nitride/Silicon Dioxide Waveguides. Opt. Express 2008, 16, 12987-12994. [CrossRef]

7. Boller, K.; van Rees, A.; Fan, Y.; Mak, J.; Lammerink, R.E.M.; Franken, C.A.A.; van der Slot, P.J.M.; Marpaung, D.A.I.; Fallnich, C.; Epping, J.P.; et al. Hybrid Integrated Semiconductor Lasers with Silicon Nitride Feedback Circuits. Photonics 2020, 7, 4. [CrossRef]

8. Feng, J.; Akimoto, R.A. Three-Dimensional Silicon Nitride Polarizing Beam Splitter. IEEE Photonics Technol. Lett. 2014, 26, 706-709. [CrossRef]

9. Herr, T.; Hartinger, K.; Riemensberger, J.; Wang, C.Y.; Gavartin, E.; Holzwarth, R.; Gorodetsky, M.L.; Kippenberg, T.J. Universal Formation Dynamics and Noise of Kerr-Frequency Combs in Microresonators. Nat. Photonics 2012, 6, 480-487. [CrossRef]

10. Pfeiffer, M.H.P.; Herkommer, C.; Liu, J.; Guo, H.; Karpov, M.; Lucas, E.; Zervas, M.; Kippenberg, T.J. Octave-Spanning Dissipative Kerr Soliton Frequency Combs in $\mathrm{Si}_{3} \mathrm{~N}_{4}$ Microresonators. Optica 2017, 4, 684-691. [CrossRef]

11. Halir, R.; Okawachi, Y.; Levy, J.S.; Foster, M.A.; Lipson, M.; Gaeta, A.L. Ultrabroadband Supercontinuum Generation in a CMOS-Compatible Platform. Opt. Lett. 2012, 37, 1685-1687. [CrossRef]

12. Tan, D.T.H.; Ikeda, K.; Sun, P.C.; Fainman, Y. Group Velocity Dispersion and Self Phase Modulation in Silicon Nitride Waveguides. Appl. Phys. Lett. 2010, 96, 611016. [CrossRef]

13. Kruckel, C.J.; Torres-Company, V.; Andrekson, P.A.; Spencer, D.T.; Bauters, J.F.; Heck, M.J.R.; Bowers, J.E. Continuous WavePumped Wavelength Conversion in Low-Loss Silicon Nitride Waveguides. Opt. Lett. 2015, 40, 875-878. [CrossRef]

14. Yao, Z.; Wan, Y.; Bu, R.; Zheng, Z. Improved Broadband Dispersion Engineering in Coupled Silicon Nitride Waveguides with a Partially Etched Gap. Appl. Opt. 2019, 58, 8007-8012. [CrossRef] [PubMed]

15. Guo, H.; Herkommer, C.; Billat, A.; Grassani, D.; Zhang, C.; Pfeiffer, M.H.P.; Weng, W.; Bres, C.; Kippenberg, T.J. Mid-infrared Frequency Comb via Coherent Dispersive Wave Generation in Silicon Nitride Nanophotonic Waveguides. Nat. Photonics 2018, 12, 496. [CrossRef]

16. Okawachi, Y.; Lamont, M.R.E.; Luke, K.; Carvalho, D.O.; Yu, M.; Lipson, M.; Gaeta, A.L. Bandwidth Shaping of MicroresonatorBased Frequency Combs via Dispersion Engineering. Opt. Lett. 2014, 39, 3535-3538. [CrossRef] [PubMed]

17. Kruckel, C.J.; Fulop, A.; Ye, Z.; Andrekson, P.A.; Torres-Company, V. Optical Bandgap Engineering in Nonlinear Silicon Nitride Waveguides. Opt. Express 2017, 25, 15370-15380. [CrossRef] [PubMed]

18. Liu, Q.; Gao, S.; Li, Z.; Xie, Y.; He, S. Dispersion Engineering of a Silicon-Nanocrystal-Based Slot Waveguide for Broadband Wavelength Conversion. Appl. Opt. 2011, 50, 1260-1265. [CrossRef]

19. Zhang, L.; Yue, Y.; Xiao-Li, Y.; Wang, J.; Beausoleil, R.G.; Willner, A.E. Flat and Low Dispersion in Highly Nonlinear Slot Waveguides. Opt. Express 2010, 18, 13187-13193. [CrossRef]

20. Sun, X.; Dai, D.; Thylén, L.; Wosinski, L. Double-Slot Hybrid Plasmonic Ring Resonator Used for Optical Sensors and Modulators. Photonics 2015, 2, 1116-1130. [CrossRef]

21. Baets, R.; Subramanian, A.Z.; Clemmen, S.; Kuyken, B.; Bienstman, P.; Le Thomas, N.; Roelkens, G.; Van Thourhout, D.; Helin, P.; Severi, S. Silicon Photonics: Silicon Nitride Versus Silicon-On-Insulator. In Proceedings of the 2016 Optical Fiber Communications Conference and Exhibition (OFC), Anaheim, CA, USA, 20-22 March 2016; p. Th3J.1.

22. Mao, S.C.; Tao, S.H.; Xu, Y.L.; Sun, X.W.; Yu, M.B.; Lo, G.Q.; Kwong, D.L. Low Propagation Loss SiN Optical Waveguide Prepared by Optimal Low-Hydrogen Module. Opt. Express 2008, 16, 20809-20816. [CrossRef] [PubMed]

23. Cheng, X.; Hong, J.; Spring, A.M.; Yokoyama, S. Fabrication of a High-Q Factor Ring Resonator Using LSCVD Deposited Si ${ }_{3} \mathrm{~N}_{4}$ Film. Opt. Mater. Express 2017, 7, 2182-2187. [CrossRef]

24. Hong, J.; Spring, A.M.; Qiu, F.; Yokoyama, S. A High Efficiency Silicon Nitride Waveguide Grating Coupler with a Multilayer Bottom Reflector. Sci. Rep. 2019, 9, 12988. [CrossRef]

25. Yin, Y.; Yin, X.; Zhang, X.; Yan, G.; Wang, Y.; Wu, Y.; An, J.; Wang, L.; Zhang, D. High-Q-Factor Silica-Based Racetrack Microring Resonators. Photonics 2021, 8, 43. [CrossRef]

26. Hansryd, J.; Andrekson, P.A.; Westlund, M.; Li, J.; Hedekvist, P. Fiber-Based Optical Parametric Amplifiers and Their Applications. IEEE J. Sel. Top. Quantum Electron. 2002, 8, 506-520. [CrossRef]

27. Gao, S.M.; Li, Z.Q.; Zhang, X.Z. Power-Attenuated Optimization for Four-Wave Mixing-Based Wavelength Conversion in Silicon Nanowire Waveguides. J. Electromagn. Wave 2010, 24, 1255-1265. [CrossRef]

28. Jia, L.; Geng, M.; Zhang, L.; Yang, L.; Chen, P.; Wang, T.; Liu, Y. Wavelength Conversion Based on Degenerate-Four-Wave-Mixing with Continuous-Wave Pumping in Silicon Nanowire Waveguide. Opt. Commun. 2009, 282, 1659-1663. [CrossRef] 
29. An, L.; Liu, H.; Sun, Q.; Huang, N.; Wang, Z. Wavelength Conversion in Highly Nonlinear Silicon-Organic Hybrid Slot Waveguides. Appl. Opt. 2014, 53, 4886-4893. [CrossRef] [PubMed]

30. Jin, B.; Yuan, J.; Yu, C.; Sang, X.; Wei, S.; Zhang, X.; Wu, Q.; Farrell, G. Efficient and Broadband Parametric Wavelength Conversion in a Vertically Etched Silicon Grating without Dispersion Engineering. Opt. Express 2014, 22, 6257-6268. [CrossRef] [PubMed] 